\title{
Evaluation of efficacy of lens extraction for intraocular pressure reduction in eyes with primary angle closure glaucoma and primary angle closure
}

\author{
Agnieszka Rozegnał-Madej, Agnieszka Wilkos-Kuc, Aleksandra Wlaź, Tomasz Żarnowski \\ Department of Diagnostics and Microsurgery of Glaucoma, Medical University of Lublin, Lublin, Poland
}

\begin{abstract}
INTRODUCTION. The purpose of the study was to evaluate the intraocular pressure (IOP) lowering effect of lens removal in eyes with primary angle closure glaucoma (PACG) and primary angle closure (PAC).

MATERIALS AND METHODS. This study was a retrospective analysis of 114 eyes of 97 patients (83 women, 14 men; mean age 69.9 years) with PACG and PAC treated with lens extraction. Outcome measures: age, gender, visual acuity, IOP reduction over time, preoperative and postoperative number of IOP-lowering medications, axial length, intraocular lens (IOL) power, requirement for additional anti-glaucoma operations, and complications. Patients were divided into three groups: 1) patients with PAC (22 eyes) with mean follow-up 12.2 months; 2) patients with PACG with a history of previous acute angle closure (AAC) (39 eyes) with mean follow-up 15.4 months; and 3) patients with PACG without a history of previous AAC (53 eyes), with mean follow-up 13.5 months. Laser iridotomy was performed in all treated eyes prior to the surgery.

RESULTS. In the group with PAC mean IOP was reduced from $42.2 \pm 15.4 \mathrm{~mm} \mathrm{Hg}$ (mean $\pm \mathrm{SD}$ ) under 2.6 IOP-lowering medications to $20.2 \pm 10.4 \mathrm{~mm} \mathrm{Hg}$ under 1.1 IOP-lowering medications (mean IOP reduction 52.1\%). In the group with PACG with a history of previous AAC mean IOP was reduced from $29.0 \pm 15.7 \mathrm{~mm} \mathrm{Hg}$ under 2.1 IOP-lowering medications to $17.0 \pm 7.5 \mathrm{~mm} \mathrm{Hg}$ under 0.7 IOP-lowering medications (mean IOP reduction 41.3\%). In the group with PACG without a history of previous AAC (53 eyes) mean IOP was reduced from $22.8 \pm 8.0 \mathrm{~mm} \mathrm{Hg}$ under 1.75 IOP-lowering medications to $18.8 \pm 7.4 \mathrm{~mm} \mathrm{Hg}$ under 1.5 IOP-lowering medications (mean IOP reduction 17.5\%). In all three groups statistically significant improvement of visual acuity as a result of surgery was observed. There were no statistically significant differences between the three groups with regard to AXL or IOL power. Intraoperative complications were noticed in eight cases, and postoperative complications were noticed in four cases. The number of anti-glaucoma medications was reduced, but 17 eyes needed additional anti-glaucoma procedures.

CONCLUSIONS. Lens extraction in eyes with PACG and PAC resulted in significant IOP reduction in all groups. The most pronounced IOP reduction was achieved in the group with PAC, where lens extraction was performed up to 60 days from AAC. Visual acuity was significantly improved as a result of surgery in all three study groups.
\end{abstract}

KEY WORDS: cataract surgery, acute angle closure, angle closure glaucoma, intraocular pressure, phacoemulsification Ophthalmol J 2017; Vol. 2, No. 1, 6-12 


\section{INTRODUCTION}

The World Health Organization ranks glauco$\mathrm{ma}$ as the second most common cause of blindness after cataract and as the leading cause of irreversible blindness in the world [1]. Although primary open-angle glaucoma (POAG) is more common, primary angle-closure glaucoma (PACG) is more likely to result in severe vision loss. By 2020, 5.3 million people worldwide will be blind because of PACG [2].

Primary angle closure (PAC) is characterised by contact between the peripheral iris and the trabecular meshwork (appositional closure) leading to an elevated IOP. This contact can ultimately result in a permanent synechial closure of the angle. PACG occurs when high IOP damages the optic nerve and leads to visual loss and, if untreated or inadequately treated, causes blindness [3]. There are multiple risk factors for angle-closure glaucoma (ACG), including hyperopia, short axial length of the eyeball, anterior chamber length of less than $2.5 \mathrm{~mm}$, inhabitant of Eskimo and Southeast Asia, age over 40 years (due to thickened lens and shallow anterior chamber depth), females (because of their tendency to have shallower anterior chamber), and a family history of ACG [4]. The standard initial ACG treatment is lowering IOP medically, which is followed by laser peripheral iridotomy (LPI) relieving pupil block, the predominant mechanism of ACG. LPI remains the cornerstone of angle-closure management; however, long-term IOP control after LPI is poor [5]. The main causes of LPI ineffectiveness are plateau iris syndrome (PIS), ciliary block, peripheral anterior synechiae (PAS) or lens disproportion. If the drainage pathway is still closed after LPI, alternative laser peripheral iridoplasty is an option. If these first-line treatments fail, glaucoma surgery (e.g. trabeculectomy) is then indicated. Glaucoma surgery may be ineffective to control the IOP, and in PACG complications are more likely to develop (such as flat anterior chamber and malignant glaucoma) than for other types of glaucoma [6].

In the 1970s, Bigger and Becker reported a lower IOP after cataract extraction in glaucoma patients [7].
Since then a new approach to the management of patients with PACG (by lens extraction) has gained interest among specialists internationally, and many investigations have shown variable IOP decrease after cataract surgery.

The aim of this study was to characterise the intraocular pressure (IOP) lowering effect of lens removal in eyes with primary angle closure glaucoma (PACG) and primary angle closure (PAC).

\section{MATERIALS AND METHODS}

This study is a retrospective analysis of 114 eyes of 97 patients (83 women, 14 men) with PACG and PAC treated by lens extraction. The effectiveness of surgeries performed in the years 2008-2013 in the Department of Ophthalmology, Medical University, in Independent Public Clinical Hospital Number 1 in Lublin, was analysed. The mean age of participants was 70 years (range 50-88 years for women and 50-83 years for men), as shown in Table 1 . Mean follow-up was 408.5 days ( \pm 37.3 SEM) (30 days -6 years).

The surgical procedure was phacoemulsification with intraocular monofocal lens implantation. Prior to the surgery, in all eyes laser iridotomy was performed and treatment with anti-glaucoma medications for IOP lowering was started.

Patients were divided into three groups (Tab. 2): - primary angle closure (PAC) (22 eyes) - with lens extraction up to 60 days from acute angle closure (AAC) (mean 23.3 days, \pm 4.5 SEM);

- PACG with a history of previous AAC (39 eyes) - documented AAC over 60 days prior to lens removal (mean 57.5 months, \pm 10.2 SEM);

- PACG without a history of previous AAC (53 eyes).

PAC was defined as iridotrabecular contact of at least $180^{\circ}$ on gonioscopy, and PACG as reproducible glaucomatous visual field defects, glaucomatous optic neuropathy, or both, and intraocular pressure (IOP) greater than $21 \mathrm{~mm} \mathrm{Hg}$ on at least one visit. AAC was defined as: 1) having at least two of these symptoms: ocular pain, nausea and/or vomiting,

\begin{tabular}{|c|c|c|c|}
\hline & Women & Men & Together \\
\hline Number of patients & $83(85.6 \%)$ & $14(14.4 \%)$ & 97 \\
\hline Number of eyes & $97(85.1 \%)$ & $17(14.9 \%)$ & 114 \\
\hline Mean age (years) ( \pm SEM) & $69.7( \pm 1.06)$ & $71.1( \pm 2.14)$ & $69.9( \pm 0.95)$ \\
\hline
\end{tabular}

SEM — standard error of the mean 


\begin{tabular}{|l|c|c|c|}
\hline Table 2. Study groups & & & \\
\hline & PAC & PACG with AAC & PACG without AAC \\
\hline Number of eyes & $22(19.3 \%)$ & $39(34.2 \%)$ & $53(46.5 \%)$ \\
\hline Mean follow-up (days) $( \pm$ SEM) & $367.5( \pm 66.9)$ & $457.3( \pm 67.6)$ & $389.6( \pm 56.9)$ \\
\hline
\end{tabular}

PAC — primary angle closure; PACG — primary angle closure glaucoma; AAC — acute angle closure; SEM — standard error of the mean

\begin{tabular}{|c|c|c|c|}
\hline & $\begin{array}{c}\text { Mean BCVA before } \\
\text { surgery ( } \pm \text { SEM) }\end{array}$ & $\begin{array}{l}\text { Mean BCVA after } \\
\text { surgery ( } \pm \text { SEM) }\end{array}$ & $\%$ improvement \\
\hline PAC & $0.25 \pm 0.05$ & $0.54 \pm 0.07^{* \star *}$ & 116.00 \\
\hline PACG with AAC & $0.45 \pm 0.05$ & $0.59 \pm 0.05^{* *}$ & 31.11 \\
\hline PACG without AAC & $0.44 \pm 0.04$ & $0.63 \pm 0.04^{* * *}$ & 43.18 \\
\hline
\end{tabular}

PAC — primary angle closure; PACG — primary angle closure glaucoma; $A A C$ — acute angle closure; SEM — standard error of the mean

and blurred vision; 2) intraocular pressure of more than $21 \mathrm{~mm} \mathrm{Hg}$ with at least three of these signs: corneal epithelial oedema, conjunctival injection, non-reactive mid-dilated pupil, and shallow anterior chamber; and 3) the presence of an occluded angle in the affected eye, which is proven by gonioscopy. Occludable angle closure was defined if the posterior trabecular meshwork could not be visualised in at least three quadrants.

The main outcome measures were age, gender, best corrected visual acuity (BCVA), intraocular pressure (IOP) before and after surgery, number of glaucoma medications before and after surgery, axial length (AXL), intraocular lens (IOL) power, additional anti-glaucoma interventions, and intraoperative and postoperative complications.

Medical data were collected from source documentation and surgical reports of each patient saved by ophthalmological specialists. BCVA results were measured with standard Snellen charts. IOP was assessed by Goldmann applanation tonometry. To determine the presence and severity of glaucomatous neuropathy, visual field and optical coherence tomography results were evaluated. Standard automated perimetry (SAP) using Humphrey Field Analyser was performed for the detection of visual field defects. Spectral-domain optical coherence tomography (SD-OCT) with Cirrus HD-OCT was used for optical disc imaging and measurements. Measurement of AXL and the IOL power calculations were performed using either IOL Master or ultrasound, depending on the severity of cataracts and transparency of optical centres. Among 114 eyes in six cases clear lens extraction was performed (three eyes in PAC group and three eyes in PACG with ACC group).
Before and after surgery anti-glaucoma drugs were used. Topical medical treatment and/or systemic acetazolamide or mannitol were administrated. In cases with uncontrolled glaucoma additional procedures were performed.

Statistical analysis was performed to assess the results. One-way analysis of variance (ANOVA) was used to determine whether there were any statistically significant differences between the means in the three study groups. Additionally, Tukey's test was used to compare and identify any differences between results greater than the expected standard error. The Wilcoxon signed-rank test was used to investigate and estimate changes in the results of BCVA, IOP, and the number of anti-glaucoma medications during total follow-up.

\section{RESULTS}

In all three groups statistically significant BCVA improvement after surgery was observed (Tab. 3). The greatest improvement (116\%) was found in the first group, where lens extraction was performed up to 60 days from AAC.

The analysis of the dataset showed statistically significant IOP decrease in all groups after cataract surgery (Tab. 4). However, the greatest reduction was found in the PAC group (52.26\%). Figure 1 presents the IOP changes in the first year of observation. Statistical analysis revealed significant differences in IOP measurements between PAC and other groups prior to lens extraction, while there were no statistically significant differences continued for an observation period of one year after surgery. Intraocular pressure (IOP) results during total follow-up are graphically shown in Figure 2. 


\begin{tabular}{|c|c|c|c|}
\hline & $\begin{array}{l}\text { Mean IOP before } \\
\text { surgery ( } \pm \text { SEM) }\end{array}$ & $\begin{array}{c}\text { Mean IOP after surgery } \\
( \pm \text { SEM) }\end{array}$ & $\%$ decrease \\
\hline PAC & $42.27 \pm 3.27$ & $20.18 \pm 2.21^{* \star *}$ & 52.26 \\
\hline PACG with AAC & $29.69 \pm 2.49$ & $17.28 \pm 1.19^{\star \star \star}$ & 41.80 \\
\hline PACG without AAC & $22.74 \pm 1.10$ & $18.28 \pm 0.93^{\star \star \star}$ & 19.61 \\
\hline
\end{tabular}

PAC - primary angle closure; PACG — primary angle closure glaucoma; AAC — acute angle closure; SEM — standard error of the mean

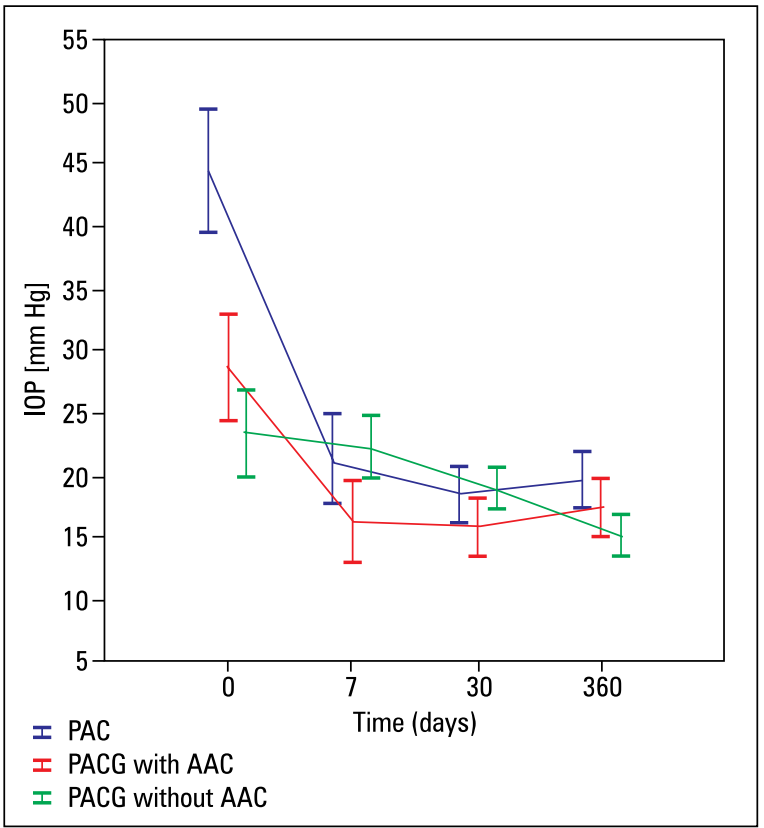

FIGURE 1. Intraocular pressure (IOP) during the first year of follow-up. Vertical bars represent \pm standard error of the mean (SEM) ANOVA: $\mathrm{F}(6,93)=3.8726, \mathrm{p}=0.00171$; Post-hoc Tukey's test showed statistically significant differences between PAC and other groups before surgery at $p<0.05$

The number of anti-glaucoma medication was also reduced, but dissimilarity between groups was noticed. Statistical analysis revealed a significant decrease in the PAC group and the PACG with AAC group, but for PACG eyes without a history of previous AAC the results were on the border of statistical significance. The largest reduction in the number of glaucoma medications were found in the group with PACG eyes, where cataract surgery was performed over 60 days after AAC (Tab. 5).

There were no statistically significant differences between the three groups with regard to axial length (AXL) or intraocular lens (IOL) power (Tab. 6).

There were intraoperative and postoperative complications noted. Posterior capsular rupture and vitreous loss were the most common intraoperative complications, found in six cases: one $(4.5 \%)$ in the PAC group , three $(7.7 \%)$ in the PACG with AAC group, and two (3.8\%) in the PACG without AAC group. During phacoemulsification in one $(1.9 \%)$ eye from the PACG without ACC group, intraoperative floppy iris syndrome (IFIS) was reported. Other intraoperative complication was Descemet's membrane detachment found in one $(2.6 \%)$ eye with PACG where lens removal was performed over 60 days after AAC. Postoperative complications were noticed in four cases. Malignant glaucoma was the most frequent postoperative complication diagnosed in one (4.5\%) eye from the PAC group and two $(3.8 \%)$ eyes from the PACG without AAC group. In the PACG without AAC group one $(1.9 \%)$ eye developed bullous keratopathy. All complications were treated in accordance with applicable standards.

Additional anti-glaucoma procedures were needed in 17 eyes. Trabeculectomy was the most frequent procedure, performed in six (11.3\%) eyes from the PACG without AAC group, two (9\%) eyes from the PAC group, and only one (2.6\%) eye from the PACG with AAC group. Vitrectomy and iridectomy with zonulectomy as a malignant glaucoma treatment was reported in two $(3.8 \%)$ eyes from the PACG without AAC group and one (4.5\%) eye from the PAC group. Other additional interventions were cyclophotocoagulation and goniosynechiolisis. $\mathrm{Cy}$ clophotocoagulation was noted in three eyes - one $(4.5 \%)$ in the PAC group and two $(5.1 \%)$ in the PACG with AAC group. Goniosynechiolisis was reported in two eyes - one (4.5\%) in the PAC group and one $(2.6 \%)$ in the PACG with AAC group.

\section{DISCUSSION}

Standard approach to the patient with primary AAC is a stepped combination of medical and laser therapy. Glaucoma surgery is indicated as a second-line option when these treatments fail. However, recently cataract extraction is more often considered for primary AAC management. 


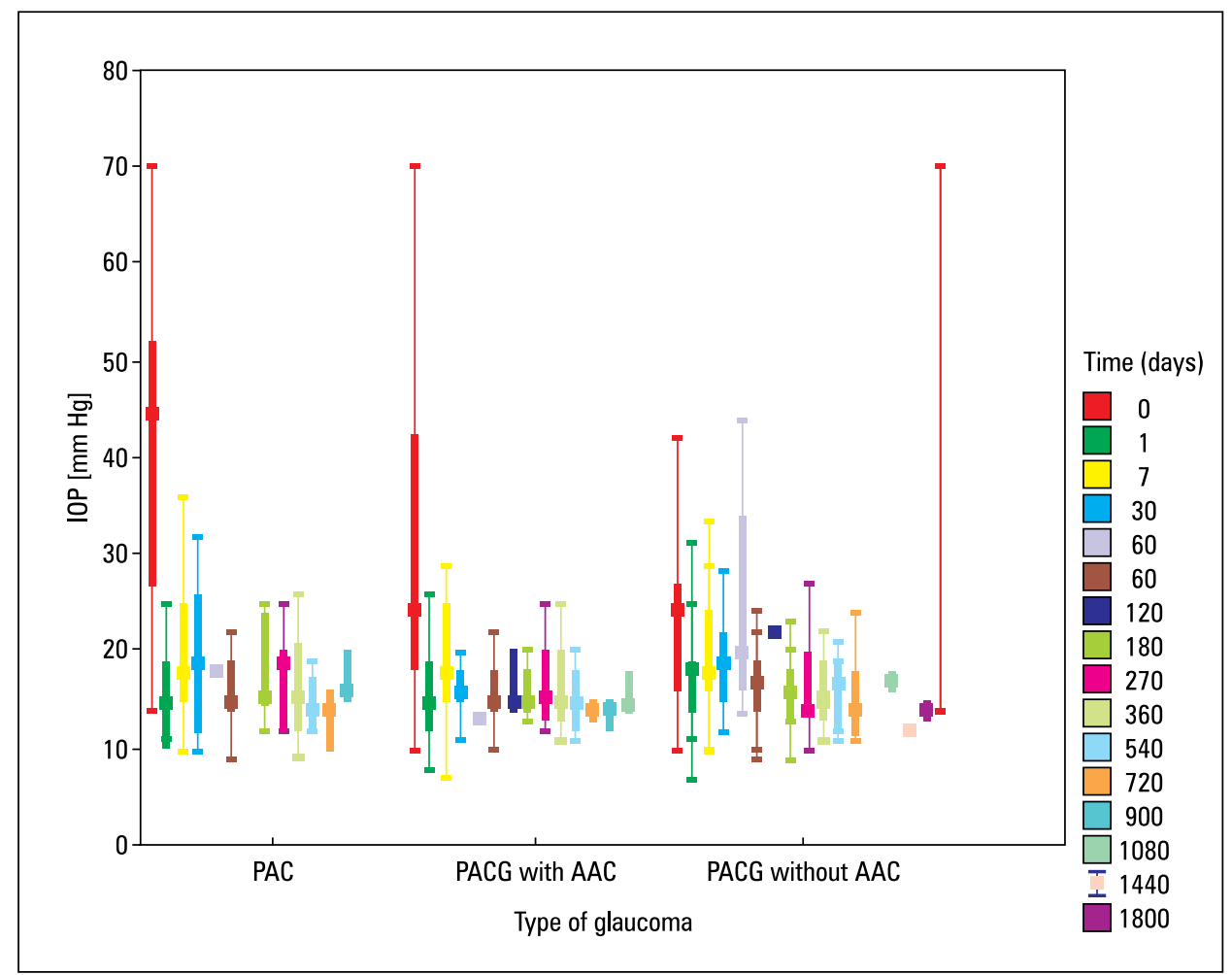

FIGURE 2. Intraocular pressure (IOP) during total follow-up; Median (percentile 25-75); whisker: non-outlier range; PAC — primary angle closure; PACG — primary angle closure glaucoma; AAC — acute angle closure

\begin{tabular}{|l|c|c|c|}
\hline \multicolumn{2}{|c|}{ Table 5. Number of glaucoma medications (Wilcoxon signed-rank test; * $p<0.05, * * p<0.01, * * * p<0.001)$} \\
\hline & $\begin{array}{c}\text { Mean number of glaucoma medications } \\
\text { before surgery ( } \pm \text { SEM) }\end{array}$ & $\begin{array}{c}\text { Mean number of glaucoma medications } \\
\text { after surgery ( } \pm \text { SEM) }\end{array}$ & $\%$ decrease \\
\hline PAC & $2.63 \pm 0.31$ & $1.09 \pm 0.25^{* *}$ & 58.55 \\
\hline PACG with AAC & $2.11 \pm 0.21$ & $0.77 \pm 0.19^{* * *}$ & 63.56 \\
\hline PACG without AAC & $1.83 \pm 0.17$ & $1.57 \pm 0.18$ & 14.43 \\
\hline
\end{tabular}

PAC — primary angle closure; PACG — primary angle closure glaucoma; AAC — acute angle closure; SEM — standard error of the mean

\section{Table 6. Axial length and intraocular lens power}

\begin{tabular}{|l|c|c|c|}
\hline & PAC & PACG with AAC & PACG without ACC \\
\hline $\begin{array}{l}\text { Mean axial length (AXL) }[\mathrm{mm}] \\
( \pm \text { SEM) }\end{array}$ & $21.9 \pm 0.19$ & $21.6 \pm 1.17$ & $21.7 \pm 0.19$ \\
\hline $\begin{array}{l}\text { Mean intraocular lens power } \\
(\text { IOL) }[\mathrm{D}]( \pm \text { SEM) }\end{array}$ & $25.8 \pm 0.44$ & $25.7 \pm 0.34$ & $25.2 \pm 0.33$ \\
\hline
\end{tabular}

'ANOVA: $F(2,103)=0.61 ; p=0.54 ;{ }^{2}$ ANOVA: $F(2,111)=0.88 ; p=0.42$

PAC — primary angle closure; PACG — primary angle closure glaucoma; AAC — acute angle closure; SEM — standard error of the mean

Pupillary block is the most common cause of ACC. Laser peripheral iridotomy is a gold standard for interrupting this configuration. However, the Liwan Eye Study showed that among eyes identified as primary closure angle suspects (PACS), LPI resulted in a significant increase in the angle width, but about one fifth of eyes had residual angle clo- sure based on gonioscopy two weeks after the LPI. Quantitative assessment of the angle using UBM demonstrated that residual angle closure tended to occur in eyes with smaller anterior chamber angle dimensions, a thicker and more anteriorly inserted iris, and a more anterior positioned ciliary body [8]. Another study demonstrated that among patients 
with persistent occludable angles, the most common underlying mechanisms were plateau iris and lens-induced component [9]. PACG eyes have significantly thicker lens, with a steeper anterior surface, which is more often anteriorly positioned when compared with controls [10]. Thick lens usually increases pupillary block and consequent angle closure. Removal of the lens allows anterior chamber deepening and angle widening, which results in increasing the humour outflow and IOP reduction. These biometrical changes are more pronounced in ACG patients than in OAG patients or the control group [11].

IOP decrease may be also associated with other mechanisms. Poley et al. suggest that lens removal allows the posterior capsule to move posteriorly, dislodging the zonula over the ciliary body with a consequent widening of Schlemm's canal and aqueous humour drainage improvement [12]. Another theory suggests that ultrasound energy used for phacoemulsification procedure is responsible for a sudden rise of pressure in the anterior chamber, producing inflammatory cytokines (mostly IL-1) that stimulate metalloproteinase production and trabecular meshwork remodelling, facilitating humour drainage [13].

Comparison of LPI and phacoemulsification revealed better long-term IOP control after resolving acute angle closure crisis with lens extraction. Further advantages were reduced need for long-term medication in order to maintain an optimal IOP, wider angle and fewer PAS formation, with a smaller risk of a second angle closure crisis or progression to chronic angle closure, which is approximately $58.1 \%$ after LPI $[5,14]$. Also, when IOP at the base line is higher than $55 \mathrm{~mm} \mathrm{Hg}$ (which is an independent risk factor of long-term uncontrolled IOP), patients are less likely to require IOP-lowering therapy if treated with early cataract extraction, when comparing with LPI [14]. Phacoemulsification is, however, a more dangerous procedure due to the risk of shallow anterior chamber, possibility of endothelium damage and atonic iris [15]. In our retrospective study eight intraoperative and four postoperative cases of complications were noted. The most frequent complication (five eyes) was posterior capsule rupture, which is also the most common complication during cataract surgery, reported with similar frequency in other studies [16]. Postoperative malignant glaucoma (diagnosed in three study eyes) is a pathological condition, the higher incidence is associated with a narrow or closed angle, shallow anterior chamber, and hyperopia [17].
When the anterior chamber angle is chronically closed by peripheral anterior synechiae (PAS) combined phacoemulsification and goniosynechialysis is a preferred method in treating ACG with concomitant cataract. It is considered as a reasonable, highly effective, and safe procedure [18]. Depending on the degree of angle closure, different approaches are recommended. Phacoemulsification alone might be more suitable for ACG eyes with angle closure $<180^{\circ}$, combined phaco-goniosynechialiysis might be more suitable for ACG eyes with angle closure of $180-270^{\circ}$, and combined phaco-trabeculectomy might be more suitable for ACG eyes with angle closure $>270^{\circ}[19]$.

Clear lens extraction in eyes with AAC is still under debate. In our retrospective study it was performed in six among 114 cases (three eyes in the PAC group and three eyes in the PACG with ACC group). With good visual acuity LPI appears to have a better risk-to-benefit ratio. Nevertheless, in selected cases, lens extraction may be a better option for IOP control. To help clarify this matter the EAGLE trial was designed. This multicentre prospective randomised clinical trial compared clear-lens extraction and standard care with LPI and topical medical treatment in patients with newly diagnosed primary angle-closure and primary angle closure glaucoma. The co-primary endpoints were patient-reported health status, intraocular pressure, and incremental cost-effectiveness ratio per quality-adjusted life-gained 36 months after treatment. The results showed that clear lens extraction had greater efficacy and was more cost-effective, especially over the long term. Significantly fewer patients in this group needed any treatment to control IOP and fewer needed glaucoma medications than patients who received standard care. The authors suggested that clear lens extraction should be considered as an option for first-line treatment [20]. Economic findings from the EAGLE study indicated that lens extraction has a $67-89 \%$ chance of being cost effective at three years (fewer subsequent procedures including repeat LPI, lens extraction or glaucoma surgery, and lower medication use), and it may be cost saving by 10 years [21].

In another study, Tham et al. confronted phacoemulsification and trabeculectomy in primary ACG patients with clear lens. Both procedures were effective in reducing IOP (reduction of $34 \%$ for phacoemulsification vs. $36 \%$ for trabeculectomy). Trabeculectomy-treated patients seemed to be less dependent on the additional anti-glaucoma medica- 
tions than phacoemulsification-treated patients, but at the same time trabeculectomy was associated with significantly more surgical complications ( 46 vs. $4 \%$ ), and cataract formation was the most frequent of them in long-term observation (33\%) [22].

\section{CONCLUSIONS}

Lens extraction in eyes with PAC and PACG resulted in significant IOP reduction in all groups. The biggest IOP reduction was achieved in the group with PAC, where lens extraction was performed up to 60 days from AAC. BCVA was significantly improved in all three groups as a result of surgery. There were no statistically significant differences between the three groups with regard to AXL or IOL power. Intraoperative complications were noticed in eight cases, and postoperative complications were noticed in four cases. The number of anti-glaucoma medications was reduced, but 17 eyes needed additional anti-glaucoma procedures.

Lens extraction by phacoemulsification with IOL implantation could be an effective method of treatment in PACG patients, but further long-term prospective studies are needed to fully evaluate their efficacy.

\section{REFERENCES}

1. Azuara-Blanco A, Burr JM, Cochran C, et al. Effectiveness in Angle-closure Glaucoma of Lens Extraction (EAGLE) Study Group. The effectiveness of early lens extraction with intraocular lens implantation for the treatment of primary angle-closure glaucoma (EAGLE): study protocol for a randomized controlled trial. Trials. 2011; 12: 133, doi: 10.1186/1745-6215-12-133, indexed in Pubmed: 21605352.

2. Quigley HA, Broman AT. The number of people with glaucoma worldwide in 2010 and 2020. Br J Ophthalmol. 2006; 90(3): 262-267, doi: 10.1136/bjo.2005.081224, indexed in Pubmed: 16488940.

3. Foster PJ, Buhrmann R, Quigley HA, et al. The definition and classification of glaucoma in prevalence surveys. Br J Ophthalmol. 2002; 86(2): 238-242, indexed in Pubmed: 11815354

4. Patel K, Patel S. Angle-closure glaucoma. Dis Mon. 2014; 60(6): 254-262, doi: 10.1016/j.disamonth.2014.03.005, indexed in Pubmed: 24906670 .

5. Husain R, Gazzard G, Aung T, et al. Initial management of acute primary angle closure: a randomized trial comparing phacoemulsification with laser peripheral iridotomy. Ophthalmology. 2012; 119(11): 2274-2281, doi: 10.1016/j.ophtha.2012.06.015, indexed in Pubmed: 22885123.

6. Aung T, Tow SL, Yap EY, et al. Trabeculectomy for acute primary angle closure. Ophthalmology. 2000; 107(7): 1298-1302, indexed in Pubmed: 10889101.

7. Mansberger SL, Gordon MO, Jampel H, et al. Ocular Hypertension Treatment Study Group. Reduction in intraocular pressure after cataract extraction: the Ocular Hypertension Treatment Study. Ophthalmology. 2012; 119(9): 1826-1831, doi: 10.1016/j.ophtha.2012.02.050, indexed in Pubmed: 22608478.
8. He M, Friedman DS, Ge J, et al. Laser peripheral iridotomy in eyes with narrow drainage angles: ultrasound biomicroscopy outcomes. The Liwan Eye Study. Ophthalmology. 2007; 114(8): 1513-1519, doi: 10.1016/j.ophtha.2006.11.032, indexed in Pubmed: 17462739.

9. Junqueira DLM, Prado VG, Lopes FS, et al. Non-pupillary block angle-closure mechanisms: a comprehensive analysis of their prevalence and treatment outcomes. Arq Bras Oftalmol. 2014; 77(6): 360-363, doi: 10.5935/0004-2749.20140090, indexed in Pubmed: 25627181.

10. Lowe RF. Anterior lens curvature. Comparisons between normal eyes and those with primary angle-closure glaucoma. $\mathrm{Br} \mathrm{J}$ Ophthalmol. 1972; 56(5): 409-413, indexed in Pubmed: 5044101.

11. Hayashi $K$, Hayashi $H$, Nakao F, et al. Effect of cataract surgery on intraocular pressure control in glaucoma patients. J Cataract Refract Surg. 2001; 27(11): 1779-1786, indexed in Pubmed: 11709251.

12. Poley BJ, Lindstrom RL, Samuelson TW, et al. Intraocular pressure reduction after phacoemulsification with intraocular lens implantation in glaucomatous and nonglaucomatous eyes: evaluation of a causal relationship between the natural lens and open-angle glaucoma. J Cataract Refract Surg. 2009; 35(11): 1946-1955, doi: 10.1016/j. jcrs.2009.05.061, indexed in Pubmed: 19878828.

13. Wang N, Chintala SK, Fini ME, et al. Ultrasound activates the TM ELAM-1/L-1/NF-kappaB response: a potential mechanism for intraocular pressure reduction after phacoemulsification. Invest Ophthalmol Vis Sci. 2003; 44(5): 1977-1981, indexed in Pubmed: 12714632.

14. Lam DSC, Leung DYL, Tham CCY, et al. Randomized trial of early phacoemulsification versus peripheral iridotomy to prevent intraocular pressure rise after acute primary angle closure. Ophthalmology. 2008; 115(7): 1134-1140, doi: 10.1016/j.ophtha.2007.10.033, indexed in Pubmed: 18164064.

15. Lachkar Y. Acute angle closure and angle closure glaucoma: Phacoemulsification as first-line treatment. J Fr Ophtalmol. 2010; 33(4): 273-278, doi: 10.1016/j.jfo.2010.02.009, indexed in Pubmed: 20347182.

16. Day AC, Donachie PHJ, Sparrow JM, et al. Royal College of Ophthalmologists' National Ophthalmology Database. The Royal College of Ophthalmologists' National Ophthalmology Database study of cataract surgery: report 1 , visual outcomes and complications. Eye (Lond). 2015; 29(4): 552-560, doi: 10.1038/eye.2015.3, indexed in Pubmed: 25679413.

17. Varma DK, Belovay GW, Tam DY, et al. Malignant glaucoma after cataract surgery. J Cataract Refract Surg. 2014; 40(11): 1843-1849, doi: 10.1016/j.jcrs.2014.02.045, indexed in Pubmed: 25248296.

18. Yu J, Sun M, Wei Y, et al. The timing of goniosynechialysis in treatment of primary angle-closure glaucoma combined with cataract. Mol Vis. 2012; 18: 1074-1082, indexed in Pubmed: 22605920.

19. Zhang ZM, Niu Q, Nie Y, et al. Reduction of intraocular pressure and improvement of vision after cataract surgeries in angle closure glaucoma with concomitant cataract patients. Int J Clin Exp Med. 2015; 8(9): 16557-16563, indexed in Pubmed: 26629184.

20. Azuara-Blanco A, Burr J, Ramsay C, et al. Effectiveness of early lens extraction for the treatment of primary angle-closure glaucoma (EAGLE): a randomised controlled trial. The Lancet. 2016; 388(10052): 1389-1397, doi: 10.1016/s0140-6736(16)30956-4.

21. Javanbakht M, Azuara-Blanco A, Burr JM, et al. Early lens extraction with intraocular lens implantation for the treatment of primary angle closure glaucoma: an economic evaluation based on data from the EAGLE trial. BMJ Open. 2017; 7(1): e013254, doi: 10.1136/bmjopen-2016-013254, indexed in Pubmed: 28087548.

22. Tham CCY, Kwong YYY, Baig N, et al. Phacoemulsification versus trabeculectomy in medically uncontrolled chronic angle-closure glaucoma without cataract. Ophthalmology. 2013; 120(1): 62-67, doi: 10.1016/j. ophtha.2012.07.021, indexed in Pubmed: 22986111. 